\title{
Globalisierungsfluten und Strafbarkeitsinseln - Ein Plädoyer für die Abschaffung des strafrechtlichen Ehrenschutzes
}

Von Wiss. Mitarbeiter Michael Kubiciel und Assessor Thomas Winter, Freiburg i. Br.

\section{Einleitung}

Staub läßt sich besonders leicht dort aufwirbeln, wo er sich über Jahre und Jahrzehnte angesammelt hat. So war es kein Wunder, daß die Rechtsprechung des Bundesverfassungsgerichts zum Verhältnis zwischen Meinungsfreiheit und Ehrenschutz Mitte der neunziger Jahre heftige oder - um im Bild zu bleiben - geradezu allergische Reaktionen hervorrief ${ }^{1}$. In der Rückschau erinnert die Debatte freilich eher an einen Sturm im Wasserglas als an ein reinigendes Gewitter, folgte diesem doch keine Aufhellung, sondern legte sich im Gegenteil schon bald nach und mit der Aufregung wieder eine erste Staubschicht auf die Beleidigungsdelikte. Dabei droht dem strafrechtlichen Ehrenschutz neues Ungemach: Angesichts einer globalen Kommunikation, wie sie das Internet ermöglicht, erweist er sich augenblicklich entweder als ohnmächtig oder überfordert, und innerhalb der eigenen Grenzen läuft ihm der zivilrechtliche Persönlichkeitsschutz, wie ihn vor allem Prominente zunehmend in Anspruch nehmen, den Rang ab. Daneben erweisen sich die alten Probleme einer unbestimmten Tatbestandsfassung und einer durch das Verfahren bedingten praktischen Bedeutungslosigkeit gegenüber jeglichen Lösungsversuchen resistent. Schließlich bleiben berechtigte Zweifel, ob ausgerechnet das Strafrecht die richtige Bühne zur Abwägung zwischen Meinungsfreiheit und Ehrenschutz bietet. Die Frage nach der Zukunft des strafrechtlichen Ehrenschutzes stellt sich also nicht als eine des Wieviel, sondern vielmehr als eine des $\mathrm{Ob}^{2}$.

i BVerfGE 93, 266; vgl. zu den Reaktionen etwa Kiesel, NVwZ 1992, 1190 („Liquidierung des Ehrenschutzes“); Schmitt Glaeser, NJW 1996, 873, 878 („unerträgliche Rohheit des Umgangs“); Sendler, NJW 1993, 2157 („Libertinage“ statt „Liberalität“, „skandalöser Unrechtszustand“); Tettinger, Die Ehre - ein ungeschütztes Rechtsgut?, 1995, S. 42 („Menschenhatz, Pranger und seelische Folter“); siehe auch die Zusammenstellung bei Kübler, NJW 1999, 1281 m.w. N.; eingehend zur Rechtsprechung des BVerfG außerdem Meurer, Festschrift für Hirsch, 1999, S. 651.

2 Vgl. dazu auch den Gesetzentwurf der Fraktion der Grünen, BT-Drucks. 11/1040, S. 7, der eine Zuordnung zum Ordnungswidrigkeitenrecht vorsah, aber nicht weiter verfolgt wurde. Zu Reformerwägungen siehe auch Hirsch, Festschrift für Wolff, 1998, S. $125,126 f$. 
Sowohl den aktuellen als auch den traditionellen Fragestellungen soll im folgenden nachgespürt werden, wobei die Ehrschutzdelikte in toto zur Disposition gestellt werden, ziehen sich die skizzierten Probleme doch durch sämtliche Normen des 14. Abschnitts des Strafgesetzbuches.

\section{Unschärfe und Bedeutungsverlust des Ehrbegriffs}

Das Dilemma des strafrechtlichen Ehrenschutzes wuchs in dem Maße, in dem die ständische Gesellschaftsordnung an Bedeutung verlor ${ }^{3}$ : Seither ist man bestrebt, mittels der Beleidigungstatbestände ein (Verfassungs-)Rechtsgut zu schützen, über das diese Tatbestände im Lichte von Art. 103 Abs. 2 GG nicht hinreichend Rechenschaft abzulegen vermögen ${ }^{4}$. Im Spannungsfeld zwischen der vollständigen Erfassung vermeintlich unerwünschter sozialer Phänomene und dem Gebot einer hinreichenden Bestimmtheit von Strafnormen haben sich sämtliche Versuche einer leistungsfähigen Begriffsbestimmung als untauglich erwiesen, da sie zwangsläufig eine Seite vernachlässigen. So sind die heute vertretenen Ehrbegriffe eher Ergebnis ihres rechtspolitischen Vorverständnisses als Maßstäbe von wissenschaftlicher Schärfe ${ }^{5}$. Dies wiegt um so schwerer, als sich sowohl der Umfang des Beleidigungsschutzes als auch der Kreis der passiv Beleidigungsfähigen und die Systematik der einzelnen Beleidigungstatbestände überzeugend nur von einem klar bestimmten Rechtsgut ableiten lassen ${ }^{6}$.

Während andere Rechtsgüter durch Anschauung oder Umschreibung in der positiven Rechtsordnung wenigstens in ihrem Kern konkretisiert sind, geht es bei der Definition der Ehre um die Festlegung eines Wertes (norma-

3 Jakobs, Festschrift für Jescheck, 1985, S. 627, 635.

4 Die Ehre ist als das den $\int S 185 \mathrm{ff}$. StGB zugrunde liegende Rechtsgut heute fast unbestritten; vgl. nur BGHSt. 1, 288, 289, BGHSt. 11, 67, 701., Herdegen, in: LK, 10. Aufll. 1989, vor $\ 185$ Rdn. 1, Lenckner, in: Schönke/Schröder, StGB, 26. Aufl. 2001, vor $\$ 185$ Rdn. 1, Rudolphi, in: SK StGB, vor $\ 185$ Rdn. 1, Tenckhoff, Die Bedeutung des Ehrbegriffs für die Systematik der Beleidigungstatbestände, 1974, S. 25, ders., JuS 1988, 199, 201, jeweils m.w.N.; anders noch Bassenge, Ehre und Beleidigung, 1937, S. 27.

5 Ebenso Ritze, JZ 1980, 91, 92.

6 Zur Notwendigkeit eines klaren Ehrbegriffs für die angesprochenen Folgen Hirsch, Festschrift für Wolff, 1998, S. 125, 150; Tenckhoff, JuS 1988, 199, $201 \mathrm{f}$.; zu den über den Ehrbegriff hinausgehenden Bestimmtheitsproblemen insbesondere des $₫ 185 \mathrm{StGB}$ Ignor, Der Straftatbestand der Beleidigung, 1995, S. $158 \mathrm{ff}$. 
tiver Ehrbegriff) oder die Erfassung eines Gefühls oder Ansehens (faktischer Ehrbegriff) ${ }^{7}$. Letzterer hat jedoch seine Bedeutung mit der Einsicht verloren, daß das Rechtsgut Ehre (und damit letztlich die Strafbarkeit) nicht von der keinem Maßstab zugänglichen Empfindlichkeit des Betroffenen abhängen kann ${ }^{8}$ und daß die Rechtsordnung nicht dazu berufen ist, mittels eines äußerlichen Ehrverständnisses den bloß tatsächlichen, aber unverdient guten Ruf zu schützen 9 .

Es bleibt die Begründung der Ehre als Wert, d.h. die Suche nach etwas, aus dem sich ein verdienter Achtungsanspruch des Menschen als Maßstab ableiten läßt, vor dem sämtliche Äußerungen über die Person Bestand haben müssen ${ }^{10}$. Aus dieser Dualität von Ehre und daraus fließendem Achtungsanspruch ${ }^{11}$ läßt sich ableiten, daß die Faktoren, die einen solchen Anspruch zu begründen vermögen, in ihrer Gesamtheit jenes Phänomen darstellen, das als strafrechtlicher Ehrbegriff benannt werden mag. Daraus folgt aber im Umkehrschluß zwingend, daß, falls sich ein Achtungsanspruch nicht begründen läßt, der Versuch einer hinreichend bestimmten strafrechtlichen Begriffsbildung für die Erscheinung Ehre scheitern muß. Die Elemente, aus denen man einen solchen Anspruch herleiten könnte, sind begrenzt. Die Literatur stützt sich vor allem auf zwei Grundlagen: Einige Autoren ziehen die Fähigkeit des Menschen zu sittlichem Handeln heran, wonach die Ehre in der "Personalität und der darauf beruhenden Personenwürde" wurzele (personaler Ehrbegriff) ${ }^{12}$; andere stellen auf die soziale Geltung und Anerkennung des einzelnen $\mathrm{ab}$, wodurch sich Inhalt und Maßstab der Ehre im

7 Auf dic Besonderheit des Rechtsguts „Ehre“ weist auch hin E. A. Wolff, ZStW 81 (1969), S. 886, 901; Übersichten zu den verschiedenen Ehrbegriffen bei Engisch, Festschrift für Lange, 1976, S. 401, 402; Otto, Festschrift für Schwinge, 1973, S. $73 \mathrm{f}$, Scbößler, Anerkennung und Beleidigung, 1997, S. $21 \mathrm{ff.,} \mathrm{Lenckner,} \mathrm{in:} \mathrm{Schönke/Schrö-}$ der, vor $\$ 185$ Rdn. 1, Tenckhoff, Ehrbegriff (Anm. 4), S. 35 ff., jeweils m. w. N.

8 Engisch, Festschrift für Lange, S. 401, 410f.; Hirsch, Ehre und Beleidigung, 1967, S.15f.; Maurach/Schroeder/Maiwald, Bes. Teil 2, 8. Aufl. 1995, S. 230, Lenckner, in: Schönke/Schröder, vor $\$ 185 \mathrm{Rdn}$. 1; unklar noch BGHSt. 11, 67, 71; dagegen eindeutig einen normativen Ehrbegriff zugrundelegend BGHSt. 36, 145, 148; vgl. zur Entwicklung des Ehrbegriffs in der Rechtsprechung auch Hirsch, Festschrift für Wolff, S. 125, $132 \mathrm{f}$.

9 Engisch, Festschrift für Lange, S. 401, 408; Hirsch (Anm. 8), S. 17 ff., Maurach/Schroeder/Maiwald, Bes. Teil 2, S. 230; Lenckner, in: Schönke/Schröder, vor $₫ 185 \mathrm{Rdn} .1$; Stark, Ehrenschutz in Deutschland, 1995, S. 30.

Hirsch (Anm. 8), S. 30; Herdegen, in: LK, vor $\$ 185$ Rdn 4.

11 Hirsch (Anm. 8), S. 32; ders., Festschrift für Wolff, S. 125, $134 \mathrm{ff} . ;$ kritisch dagegen bereits Jakobs, Festschrift für Jescheck, S. 631.

12 Hirsch (Anm. 8), S. 53 ff.; Herdegen, in: LK, vor $\$ 185$ Rdn. 4. 
Verhältnis des Individuums zur Gemeinschaft konkretisieren (sozialer Ehrbegriff) ${ }^{13}$. Überzeugen können letztlich beide nicht.

Der personale Ehrbegriff zieht sich zur Bestimmung des Rechtsguts auf die Maßstäbe der Individual- und Sozialethik, der allgemeinen sittlichen Ordnung und der Situationsethik zurück ${ }^{14}$. Damit wäre an Bestimmtheit nur etwas gewonnen, wenn zwei Bedingungen erfüllt wären: In der Gesellschaft müßte es tatsächlich einen formulierbaren Konsens über die Maßstäbe der Individual- und Sozialethik geben, und diese Vorstellung müßte wenigstens in ihrem Kern statisch sein. In einer pluralistischen Gesellschaft liegen beide Voraussetzungen nicht vor. Schon ein vermeintlicher Grundkonsens in bezug auf die sittliche Ordnung und moralisches Handeln besteht immer nur aus der Sicht des Interpreten. Subjektive Empfindungen wie das Ehrgefühl sind dezentral verteilt und derart unterschiedlich, daß jede Objektivierung durch einen Dritten eine Anmaßung von Wissen bedeutet ${ }^{15}$. Außerdem bewirkt die ungeheure Dynamik einer modernen und zunehmend globalen Gesellschaft mit ihren sich schnell verändernden Anforderungen und Bewertungen menschlicher Existenz einen kontinuierlichen Wertewandel ${ }^{16}$. Diese Veränderungen in sich aufzunehmen ist natürlich auch die Rechtsordnung aufgerufen; dem Strafrecht muß es allerdings wegen seines besonders strengen Maßstabs der Voraussehbarkeit und Bestimmtheit verbotenen Handelns versagt sein, seine Begrifflichkeit anhand wechselnder und wechselhafter Anschauungen stets neu zu definieren ${ }^{17}$. Es liegt der Verdacht nahe, daß sich hinter dem Begriff der „sittlichen Ordnung" eine Leerformel verbirgt, die von ihren Verwendern unter dem Deckmantel der Wissenschaftlichkeit mit den eigenen oder den opportunen Wertüberzeugungen der jeweiligen Epoche gefüllt wird ${ }^{18}$.

Kritik verdient der personale Ehrbegriff auch, soweit versucht wird, die "Fälle elementarer menschlicher Unzulänglichkeit" 19 in ihn aufzuneh-

13 Dezidiert in diesem Sinne - auch in bewußter Unterscheidung zu Hirsch - E. A. Wolff, ZStW 81 (1969), S. 886, 901 ff.; vgl. auch Maurach/Schroeder/Maiwald, Bes. Teil 2, S. 231, Lenckner, in: Schönke/Schröder, vor $\$ 185 \mathrm{Rdn}$. 1; ausdrücklich offengelassen in BGHSt. 36, 145, 148.

14 Hirsch, Festschrift für Wolff, S. 125, 136ff., Herdegen, in: LK,vor $\ 185$ Rdn. 14.

15 Vgl. von Hayek, Recht, Gesetzgebung und Freiheit, Bd. 2, 1981, S. 23.

16 Herdegen, in: LK, vor $₫ 185$ Rdn. 14.

17 So weist Schubarth, JuS 1981, 726, 728, darauf hin, daß ein Konsens über den Inhalt der Beleidigung noch bei Erlaß des StGB bestanden haben mag, heute aber eine „communis opinio" darüber gerade fehle; anders Küpper, ZRP 1991, 249, 250.

18 Vgl. zu diesem Methodenproblem Rüthers, Rechtstheorie, 1999, S. 502 ff., 543.

19 Hirsch (Anm. 8), S. 82; Herdegen, in: LK, vor $₫ 185$ Rdn. 17. 
men ${ }^{20}$. Körperliche und geistige Mängel lassen sich nämlich nicht als schuldhafte Verstöße gegen sittliche und moralische Pflichten erfassen, weshalb der Maßstab des nur sittlichen Handelns als zu eng angesehen wird ${ }^{21}$. Um den Ansatz, die Ehre wurzele in der Personenwürde, nicht aufgeben zu müssen, wird behauptet, geistige und körperliche Gebrechen hinderten die Entstehung oder das Fortbestehen voller Personalität ${ }^{22}$, beziehungsweise es gehe um einen „die volle Achtungswürdigkeit als Person, die vollentwickelte Personenwürde vermindernden Personendefekt " ${ }^{23}$. Setzt diese Begründung aber nicht im Umkehrschluß eine mögliche Abstufung der Würde einer Person nach ihren geistigen und körperlichen Fähigkeiten voraus, welche die Würde des Menschen, wie sie in Art. 1 GG geschützt wird, gerade verbietet $^{24}$ ? Ein streng auf die Person abstellender Ehrbegriff kann geistige und körperliche Mängel nicht in sich aufnehmen. Dies wird besonders deutlich, wenn letztlich nur "der, Volksgeist', [der] erhaben über akademische Analysen des Ehrbegriffs, weiß, daß in der Zuweisung von Borniertheit eine Mißachtung liegen kann, die eines moralisierenden Elements nicht bedarf, um tatbestandsmäßig zu sein" ${ }^{25}$, zur Begründung bemüht werden kann. Offenbar gelingt es nicht, ohne Rückgriff auf zweifelhafte metaphysische Topoi und Vermutungen eine tragfähige Umschreibung dessen herauszuarbeiten, was Rechtsgut und damit ratio legis der $\$ \int 185 \mathrm{ff}$. StGB sein soll.

Der soziale Ehrbegriff findet seine Basis ebenfalls in der Personenwürde ${ }^{26}$, erweitert den Wertbezug aber um die verdiente Anerkennung des einzelnen durch andere und bezieht so die soziale Geltung der Person mit $e^{2}{ }^{27}$. Es handelt sich also um den „Versuch, die Ehre aus dem Verhältnis des

Diese Kritik gilt insoweit auch für den sozialen Ehrbegriff, da sich dieser bei in der Person liegenden Defekten nicht vom personalen Ehrbegriff unterscheidet, sondern lediglich durch die Aufnahme gegenüber der außerhalb der Person liegenden Anerkennung von Leistungen durch die Gemeinschaft; vgl. auf der Grundlage des sozialen Ehrbegriffs Maurach/Schroeder/Maiwald, Bes. Teil 2, S. 232.

21 Hirsch (Anm. 8), S. 82 ff.; Herdegen, in: LK, vor $\ 185 \mathrm{Rdn} .17$; anders und insoweit konsequent noch Binding, Lehrbuch des gemeinen deutschen Strafrechts, Besonderer Teil I, 2. Aufl. 1902, S. 144 .

22 Herdegen, in: $\mathrm{LK}$, vor $\$ 185 \mathrm{Rdn} .17$.

23 Hirsch (Anm. 8), S. 84.

24 Zwar mag in der Lebenswirklichkeit der Achtungsanspruch zunehmend von äußerlicher Vollkommenheit abhängen, doch kann vor einer Relativierung der normativ absoluten Wertsetzung durch Art. $1 \mathrm{GG}$ nur gewarnt werden. So Herdegen, in: LK, vor $\$ 185$ Rdn. 17.

26 Otto, Festschrift für Schwinge, S. 71, 75.

27 In diesem Sinne die wohl herrschende Meinung in der Literatur; vgl. Maurach/Schroeder/Maiwald, Bes. Teil 2, S. 231, Lenckner, in: Schönke/Schröder, vor $\$ 185$ Rdn. 1; 
einzelnen zur Gesellschaft zu erschließen “28. Seinen Ausgangspunkt nimmt dieser Versuch bei E. A. Wolff in der philosophisch abgeleiteten These, der einzelne allein könne seine Entwicklung „zum Freien (d. h. zum selbständigen Subjekt überhaupt)" nicht leisten und in der Folge den einmal erreichten "Status“ als solchen auch nicht allein durchhalten ${ }^{29}$. Daraus folgt die Überlegung, daß die Selbständigkeit des Menschen erst mit der Anerkennung durch andere möglich werde ${ }^{30}$. Dieses „die Selbständigkeit ermöglichende Anerkennungsverhältnis" könne rechtlich als Ehre definiert werden ${ }^{31}$. Damit gewähre die Ehre nicht mehr nur den Anspruch der Person, als Person geachtet zu werden, sondern auch „nach ihren auf die Gemeinschaft bezogenen Werken eingeschätzt zu werden " 32 . Der Ehrbegriff öffnet sich so für die Anerkennung, die einer Person verdientermaßen zuteil wird, weshalb zum Beispiel die soziale Rolle oder die erworbenen Verdienste einen Teil der Ehre bilden ${ }^{33}$.

Mußte schon dem personalen Ehrbegriff seine nicht akzeptable Unbestimmtheit vorgeworfen werden, so überschreitet der soziale Ehrbegriff die Schwelle des Bestimmtheitsgebots erst recht ${ }^{34}$. Zusätzlich zu einem sittlichen Maßstab umfaßt das Ehrverständnis hier den sozialen Geltungswert einer Person, also ihr Ansehen (sei es auch nur das verdiente - doch wer wollte über dieses Merkmal mit Verbindlichkeit entscheiden?), ihre Autorität und ihr Prestige. Das Rechtsgut der Ehre wird über einen weiten Bereich der Personalität erstreckt und mündet letztlich in eine Gleichsetzung mit dem allgemeinen Persönlichkeitsrecht, das sich einer klaren Abgrenzung entzieht und sich - unbeschadet seiner zivilrechtlichen Sinnhaftigkeit - in einer jedenfalls strafrechtlich nicht erlaubten Weite und Offenheit verliert ${ }^{35}$.

grundlegend E.A. Wolff, ZStW 81 (1969), S. 886, $893 \mathrm{ff}$.; an diesen anschließend Otto, Festschrift für Schwinge, S. 71, $74 \mathrm{ff}$.

Jakobs, Festschrift für Jescheck, S. 632, unter Hinweis auf die in der Tradition Hegels stehenden Ausführungen von E. A. Wolff, ZStW 81 (1969), S. $886 \mathrm{ff}$.

23 E. A. Wolfff, ZStW 81 (1969), S. 886, 894 .

30 E. A. Wolff, ZStW 81 (1969), S. 886, 896.

31 E. A. Wolff, ZStW 81 (1969), S. 886, 901.

32 Otto, Festschrift für Schwinge, S. 82.

33 Maurach/Schroeder/Maiwald, Bes. Teil 2, S. 232.

34 Hirsch (Anm. 8), S. 61; kritisch auch Herdegen, in: LK, vor $\ 185$ Rdn. 10f.; Jakobs, Festschrift für Jescheck, S. 632 (der von einem "geradezu ungeheuer weiten“ Angriffsobjekt schreibt); sämtliche Ehrdefinitionen lehnen als zu unbestimmt ab Findeisen/ Hoepner/Zünkler, ZRP 1991, 245, 246.

35

Hirsch (Anm. 8), S. 60f.; ders. Festschrift für Wolff, S. 125, 138; Herdegen, in: LK, vor $\$ 185$ Rdn. 2, 16; vgl. auch Gallas, ZStW 75 (1963), S. 17, 38 f. 
Richtig an der sozialen Ehrtheorie ist lediglich die ihr zugrunde liegende Analyse, daß ein wirksamer Persönlichkeitsschutz die Stellung des einzelnen in der Gesellschaft und vor allem in und gegenüber den Medien notwendig berücksichtigen muß. Die Aufnahme dieser Elemente in das Rechtsgut der Beleidigungsdelikte scheitert aber - zumindest de lege lata - an Art. 103 Abs. 2 GG $^{36}$.

Dem Bestimmmtheitsgebot genügen könnte nur ein Ehrbegriff, der auf Maßstäbe zurückgeführt werden kann, die auch in einem freiheitlichen Rechtsstaat verbindlich sind. Als solcher Maßstab kommt die positive Rechtsordnung in Betracht. Die Ehre ließe sich dann als die Fähigkeit des Menschen zu rechtlichem Handeln definieren. Daraus könnte aber für den einzelnen ausschließlich der Anspruch abgeleitet werden, nicht eines gegen die Rechtsordnung gerichteten Verhaltens geziehen zu werden. Ob ein solcher Schutz aber über den Umfang des $₫ 164$ StGB hinaus notwendig ist, kann bezweifelt werden.

Bereits die Überlegungen zum Rechtsgut der Beleidigungsdelikte zeigen, daß der Weg eines strafrechtlichen Ehren- oder gar Persönlichkeitsschutzes eine Sackgasse ist. Das Strafrecht kann den Spagat zwischen Bestimmtheitsgebot und Persönlichkeitsschutz nicht aushalten. Man sollte schon aus diesem Grund die „hölzernen Handschuhe " 37 beiseite legen und den sensiblen Schutz der Persönlichkeit mit dem eleganten, gleichwohl scharfen Florett anderer Disziplinen ausfechten.

36 Daher erweist sich auch der Verweis auf eine über 100jährige Rechtsprechung der Strafgerichte als wenig stichhaltig (so aber pauschal BVerfGE 93, 266, $291 \mathrm{f}$.); allein die Tradition verbürgt keine Bestimmtheit. Es ist der Rechtsprechung nicht gelungen, cin vor Art. 5 Abs. $1 \mathrm{GG}$ bestandskräftiges und bestimmbares Rechtsgut herauszuarbeiten, was nicht zuletzt in der Vielzahl von Verfahren vor dem Bundesverfassungsgericht sichtbar geworden ist. Im Gegenteil zeichnen sich gerade die Definitionsbemühungen des BGH durch eine generalklauselartige Weite aus, die jede Vorhersehbarkeit ausschließen. Allein die Kasuistik läßt erschreckende Rückschlüsse zu: Welche Gemeinsamkeit kann einem Ehrverständnis zugrunde liegen, das die Bezeichnungen als "Schwuler" (KG NStZ 1992, 385) oder "Jude“ (BGHSt. 8, 325) als ebenso beleidigend und damit ehrmindernd ansieht wie die Benennung als „Folterknecht“, „Henker" und „KZ-Aufseher" (BGHSt. 36, 83) oder das Ansinnen des Geschlechtsverkehrs gegen Entgelt (BGH NStZ 1992, 33)? Vgl. zur Kasuistik auch Tröndle/Fischer, StGB, 50. Aufl. 2001, \185 Rdn. 8f., und - amüsanter - Hobnel, „Doppelvogel“ und andere Beleidigungen, 1997.

37 Das Bild geht zurück auf Maurach, Bes. Teil, 5. Aufl. 1969, S. 130. 


\section{Praktische Bedeutungslosigkeit strafrechtlichen Ehrenschutzes}

Um der Kritik an „unerträglichen Strafbarkeitslücken“ durch den befürworteten Verzicht auf die Beleidigungstatbestände zuvorzukommen, ist zunächst an die bereits aktuell bestehende praktische Bedeutungslosigkeit der Ehrdelikte zu erinnern ${ }^{38}$. Sie erklärt sich durch die verfahrensrechtliche $\mathrm{Ge}-$ staltung der $\int \mathbb{S} 185 \mathrm{ff}$. StGB als Antrags- und Privatklagedelikte, welche die differenzierte materiell-rechtliche Regelung konterkariert. Entscheidend geschwächt wird die Stellung des Verletzten dabei vor allem durch die Ausgestaltung der Beleidigungsdelikte als Privatklagedelikte ${ }^{39}$ : Der Privatkläger trägt das volle Kostenrisiko des Strafverfahrens, ist als Laie mit der Prozeßführung ohne anwaltliche Unterstützung zumeist überfordert und kann nicht einmal ein Urteil erzwingen, wenn das Gericht das Verfahren - wie es in $90 \%$ der Fälle geschieht - wegen Geringfügigkeit einstellt ${ }^{40}$. Es kommt hinzu, daß der Privatklage regelmäßig ein Sühneversuch gemäß $\ 380$ StPO vorauszugehen hat, in dem sich etwa ein Drittel der Fälle durch Vergleich erledigt ${ }^{41}$.

Die unbefriedigende Stellung des Privatklägers legt zwei unterschiedliche Folgerungen nahe: Entweder die Privatklage wird aufgegeben und die Beleidigungsdelikte werden in das herkömmliche Strafverfahren integriert ${ }^{42}$, oder der strafrechtliche Ehrenschutz tritt ganz hinter den zivil- und medienrechtlichen zurück, und der Verletzte sucht seinen Rechtsschutz im Zivilprozeß. In der Konsequenz des Anliegens des Gesetzgebers und der Entwicklung des Persönlichkeitsschutzes liegt die zweite Alternative. Bereits in den Motiven zur Strafprozeßordnung von 1877 findet sich der Hinweis, daß

Vgl. dazu Arzt, JuS 1982, 717, 723 ff., der von einer „Verkümmerung des strafrechtlichen Ehrenschutzes" schreibt; Bemmann, Festschrift für Wolff, 1998, S. 33 f.; Dreher, Festschrift für Welzel, 1974, S. 917 („einem Delikt, das alltäglich unzählige Male begangen wird und gewiß eine der größten Dunkelziffern aufweist“); Otto, Festschrift für Schwinge, S. $72 \mathrm{f}$.

Arzt, JuS 1982, 717, 724; Bemmann, Festschrift für Wolff, S. 33 f.; Hirsch, Festschrift für Lange, 1976, S. 815; vgl. zur Privatklage im Stratprozeis auch Grebing, GA 1984, 1; Roxin, Strafverfahrensrecht, 25. Aufl. 1998, S. 491 ff., m. w. N. Dagegen bringt die Ausgestaltung als Antragsdelikt dem Verletzten zunächst eher Vorteile, aber als „Bagatellund Antragsdelikte teilen die $\int \mathbb{S} 185 \mathrm{ff}$. die allgemeine kriminalpolitische Misere der Bekämpfung der Kleinkriminalität"; Arzt, a. a. O., S. 723.

40 Vgl. Arzt, JuS 1982, 717, 724; Bemmann, Festschrift für Wolff, S. 33, 34; Doering, Beleidigung und Privatklage, 1971, S. 118f.; Hirsch, Festschrift für Lange, S. $815 \mathrm{f}$., m.w. N.; Koewius, Die Rechtswirklichkeit der Privatklage, 1974; von Lippa, Der Ehrenschutz im deutschen Strafrecht, 1966, S. 129.

41 Hirsch, Festschrift für Lange, S. 815, 816.

42 Dagegen zu Recht Hirsch, Festschrift für Lange, S. 815, 818. 
das Bedürfnis für einen strafrechtlichen Schutz der Ehre wenig ausgeprägt ist: „Beleidigungen und leichte Mißhandlungen sind alltägliche Vorkommnisse; sie berühren das allgemeine Wohl der bürgerlichen Gesellschaft meistens wenig, und selbst für die Beteiligten haben sie in der Regel eine viel zu geringe Bedeutung, als daß ein rechtliches oder sittliches Bedürfnis vorläge, stets eine Bestrafung herbeizuführen. Darum bildet erfahrungsgemäß die Verfolgung und die Bestrafung jener Gesetzesverletzungen nicht die Regel, sondern die Ausnahme. " ${ }^{43}$ Dieser Einschätzung entspricht die tatsächliche Erfahrung. Rohe (sprachliche) Umgangsformen und verbale Umweltverschmutzung verpuffen ohne strafrechtliche Ahndung. Die $\$ \int 185 \mathrm{ff}$. StGB entfalten weder präventive Wirkung, noch können sie einem etwaigen Vergeltungswunsch des Betroffenen gerecht werden ${ }^{44}$. Die Beleidigung ist also auch von den Strafzwecken her zur weitgehend wirkungslosen Symbolik verkommen ${ }^{45}$. Nur in den Fällen einer gewissen Macht des Opfers, sei sie durch das Ansehen der Person in der Öffentlichkeit oder durch ihre Funktion als Vertreter des Staates begründet, kommt es zur strafrechtlichen Sanktionierung ${ }^{46}$. Diese Ungleichbehandlung wäre nur zu rechtfertigen, wenn die Beleidigungsdelikte über den Schutz der persönlichen Ehre hinaus dazu berufen wären, die Autorität von Personen zu wahren, die in der Öffentlichkeit stehen oder staatliche Funktionen wahrnehmen. Eine solche Interpretation würde die $\$ \mathbb{S} 185 \mathrm{ff}$. StGB aber vollends im Unbestimmten verschwimmen lassen und ist schon aus diesem Grund abzulehnen. Die strafrechtlichen Verfahren wegen Beleidigung erweisen sich daher nicht nur als zufällig, sondern auch als unter Gleichheitsgesichtspunkten fragwürdig. Es haftet ihnen das Stigma der Ungerechtigkeit an.

Diesem letzten Vorwurf sieht sich gelegentlich auch ein ausschließlich mit zivilrechtlichen Mitteln gewährleisteter Ehrenschutz ausgesetzt ${ }^{47}$. Nur

43 Habn (Hrsg.), Materialien zur Strafprozeßordnung, 2. Aufl. 1885, S. 277; zur Geschichte und Entwicklung der Privatklage vgl. Grebing, GA 1984, 1, $2 \mathrm{ff}$.

44 Auf diese Problematik im Zusammenhang mit den Privatklagedelikten weist hin Jung, ZStW 93 (1981), S. 1147, 1167: „Durch die vergleichsweise geringe Verurteilungsquote gerät vielmehr auch die generalpräventive Wirkung der Strafandrohung selbst in Gefahr."

45 Ebenso Gounalakis/Rösler, Ehre, Meinung und Chancengleichheit im Kommunikationsprozeß, 1998, S. 86.

46 Daher sind die Ehrdelikte als „kleine Staatsschutzdelikte“ bezeichnet worden; vgl. Arzt, JuS 1982, 717, 727; Findeisen/Hoepner/Zünkler, ZRP 1991, 245.

$47 \mathrm{Vgl}$. dazu und zu weiteren Einwänden gegen eine vollständige Verlagerung des Ehrenschutzes in das Zivilrecht Hirsch, Festschrift für Engisch, 1969, S. 304; ders., Festschrift für Lange, S. 815, $820 \mathrm{ff}$. 
wer im Blickpunkt einer breiten Öffentlichkeit stehe und über ausreichende finanzielle Möglichkeiten verfüge, beschreite den Weg zu den Zivilgerichten, während der Rechtsschutz der „kleinen Leute“ auf der Strecke bleibe ${ }^{48}$. Der jedem gebührende Ehrenschutz werde so zu einem Prominentenschutz verkürzt. Insbesondere Hirsch hat daher nach einem „dritten Weg“ gesucht und vorgeschlagen, die Privatklagedelikte zwar aus dem Kriminalstrafrecht und dem Kriminalstrafprozeß herauszunehmen, sie jedoch bußgeldbewehrt als sogenannte „Verfehlungen“ ähnlich den Ordnungswidrigkeiten sowohl materiell-rechtlich als auch verfahrensrechtlich neu zu regeln ${ }^{49}$. Diesem Vorschlag liegt zwar ein zutreffender Befund zugrunde, die daraus abgeleitete Konsequenz kann jedoch hinsichtlich der Beleidigungstatbestände kaum überzeugen, bleibt sie doch ohne Not auf halbem Wege stehen ${ }^{50}$. Sie sieht sich letztlich den gleichen Einwänden ausgesetzt wie die strafrechtliche Regelung; insbesondere widerstreitet der Gedanke, daß staatliche Sanktionierung die ultima ratio sein sollte, jeglicher gesetzlichen Pönalisierung, mag sie auch nicht Straftat, sondern Verfehlung heißen. Der Schutz der Ehre - besser der Persönlichkeit - ist die private Angelegenheit des einzelnen ${ }^{51}$. Steht die Ehre im Streit und glaubt der Verletzte, ihre Wahrung verlange nicht nur nach sozialer, sondern nach rechtlicher Reaktion, so bietet ihm das Zivilrecht unabhängig von seiner Stellung in der Gesellschaft den dafür notwendigen Rahmen. Ob er von dieser Möglichkeit angesichts der dazu notwendigen Eigeninitiative und der drohenden Kosten Gebrauch macht, bleibt ihm überlassen. In der Beschränkung auf das Zivilverfahren findet der staatliche Ehrenschutz seine notwendige, aber auch hinreichende Funktion ${ }^{52}$. Denkt man die Entkriminalisierungstendenz der Privatklage konsequent zu Ende, so gelangt man zur Abschaffung der Beleidigungstatbestände ${ }^{53}$. Dagegen

48 Hirsch, Festschrift für Lange, S. 815, 820f., 827; vgl. auch ders., bei J. Meyer, ZStW 83 (1971), S. 277, 278.

49 Hirsch, ZStW 83 (1971), S. 139, 146f., 176; ders., Festschrift für Lange, S. 815, 826ff.; ähnlich bereits Krümpelmann, Die Bagatelldelikte, 1966, S. 241, der die Kategorie der „Übertretungen“ vorschlägt.

50 Grundsätzliche Kritik an der Schaffung bzw. Wiederbelebung neuer Deliktsformen bei Dreher, Festschrift für Welzel, S. 917, 926 ff.; Vogler, ZStW 90 (1978), S. 132, $153 \mathrm{ff}$.

51 Dieser Gedanke des „minima non curat praetor“ liegt schließlich auch den Privatklagedelikten zugrunde; vgl. Grebing, GA 1984, 1, 4.

52 Vgl. zu diesem Ansatz unten V. Im übrigen kommt die strafrechtliche Sanktion, soweit sie Ehrverletzungen durch die Medien ahnden will, auch zu spät; so ausdrücklich BVerfG NJW 1983, 1179.

53 Vgl. - allerdings weniger weitgehend - Stürner, JZ 1994, 865, 875: „Der historische Trend zur Entkriminalisierung des Medienpersönlichkeitsrechts verdient weitere Förderung." 
muß der dem geltenden Recht entsprechende Kompromiß zwischen einer schwachen Stellung des Betroffenen im Privatklageverfahren und dem Festhalten am Konzept strafrechtlichen Ehrenschutzes notwendig scheitern.

\section{Die Ehrschutzdelikte als Ort verfassungsrechtlicher Spannungen und diskriminierender Rechtsanwendung}

Stellt man die Ehrdelikte zur Disposition, so erhebt sich die Frage, wie sich das Grundgesetz und insbesondere die Grundrechtsgewährleistungen zu dem Fehlen eines strafrechtlichen Ehrenschutzes verhalten, insbesondere welche Rolle die $\mathbb{S} 185 \mathrm{ff}$. StGB im Grundrechtssystem einnehmen und wie sie entstehende Grundrechtsspannungen aufzulösen vermögen.

Die Beleidigungstatbestände fungieren als Schrankengesetze gegenüber verschiedenen Grundrechten und zeichnen im Zusammenspiel mit $\ 193$ StGB insbesondere das grundrechtliche Spannungsfeld zwischen der Meinungsfreiheit und dem im allgemeinen Persönlichkeitsrecht des Art. 2 Abs. 1 i. V.m. Art. 1 Abs. 1 GG verorteten Ehrenschutz nach. Zwar scheinen grobe Beleidigungen schon vorab aus dem Schutzbereich der Meinungsfreiheit auszuscheiden und erscheint die Relevanz von Art. 5 Abs. 1 GG zudem auf den Tatbestand der Beleidigung begrenzt ${ }^{54}$. Doch zeitigt bei näherer Betrachtung die Gewährleistung der Meinungsäußerungsfreiheit umfassende Wirkungen: Einmal sind die Schutzbereiche der Freiheitsrechte eher zu weit als zu eng auszulegen, so daß - auch für Art. 5 Abs. 1 GG - Kollisionen durch Abwägung und nicht durch Verdrängung eines Freiheitsinteresses zu bewältigen sind ${ }^{55}$. Vor diesem Hintergrund überrascht es nicht, daß grundsätzlich auch die Schmähkritik vom Schutzbereich des Art. 5 Abs. 1 GG umfaßt sein soll, wenngleich das Recht der Meinungsäußerung - nicht zuletzt wegen der Berührung der Menschenwürde - in diesem Fall regelmäßig hinter dem Persönlichkeitsrecht des Angegriffenen zurücktritt ${ }^{56}$. Andererseits werden falsche Tatsachenbehauptungen von vielen - allerdings in nicht konsistenter Weise - schon aus dem Schutzbereich von Art. 5 Abs. 1 GG ausgesondert, so daß sich verfassungsrechtliche Kollisionen im Rahmen

54 In diesem Sinne wohl Meurer, Festschrift für Hirsch, S. 663.

55 Allgemein dazu Murswiek, in: Sachs, GG, Art. 2 Rdn. 53; für Art. 5 Abs. 1 BGH NJW 1981, 2117, 2119.

56 Siehe nur BVerfGE 82, 272, 283 f.; 85, 1, 16; 93, 246, 292 ff.; Bethge, in: Sachs, GG, Art. 5 Rdn. 33; Leibholz/Rink/Hesselberger, GG, Stand 1999, Art. 5 Rdn. 1001. 
der $\int \mathbb{1 8 6 f . ~ S t G B}$ eigentlich nicht ergeben dürften ${ }^{57}$. Doch hat das Bundesverfassungsgericht aufgrund der Einsicht, daß sich Meinung und Tatsachenbehauptung kaum voneinander trennen lassen, den Schutzumfang von Art. 5 Abs. 1 GG erweitert und bezieht "Gemengelagen" $z$ wischen Äußerungen, die einem Beweis zugänglich sind, und Elementen des Dafürhaltens und Meinens in den Schutzbereich der Meinungsfreiheit ein ${ }^{58}$. Daher strahlt die Schutzwirkung von Art. 5 Abs. 1 GG auf sämtliche Ehrschutztatbestände aus; dem entspricht im übrigen die Auffassung, wonach der aus Art. 5 GG abzuleitende $\ 193$ StGB grundsätzlich für alle Formen der Beleidigung des 14. Abschnitts gilt ${ }^{59}$.

In stärkerem Maße als bei anderen Strafnormen ergeben sich für die Ehrschutzdelikte aufgrund ihres vagen und grundrechtlichen Spannungen ausgesetzten Rechtsguts verfassungsrechtliche Probleme ${ }^{60}$, die eine erneute Gewichtung der betroffenen Grundrechte bei der Anwendung auf den Einzelfall notwendig machen ${ }^{61}$. Der historische Strafgesetzgeber war für das Gewicht der Meinungsfreiheit noch nicht in hinreichendem Maße sensibilisiert, während der nachkonstitutionelle Reformgesetzgeber die Ehrschutzdelikte in ihrer Form beließ und damit große Verantwortung auf die Gesetzesanwender übertrug. Beides ist nur solange unproblematisch, wie sich das Gesellschaftssystem auf tradierte und festzementierte gesellschaftliche Rollen stützt, die eines besonderen staatlichen Schutzes bedürfen, soweit sie sich ihrer Behauptung in einem pluralistischen Meinungsaustausch nicht sicher sein können ${ }^{62}$.

BVerfGE 54, 208, 219; Bethge, in: Sachs, GG, Art. 5 Rdn. 28 m. w. N.; anders Wendt, in: von Münch/Kunig (Hrsg.), GG, Bd. 1, 4. Aufl. 1992, Art. 5 Rdn. 10 m. w. N., der den gesamten thematischen Schutzbereich des Art. 5 Abs. 1 Satz 1 GG von einer Bewertung nach Inhalt, Wert oder Unwert freistellen will und eine Lösung im Bereich der Abwägung vorschlägt; ähnlich Leibholz/Rink/Hesselberger (Anm. 56), Art. 5 Rdn. 690. Auch die Rechtsprechung schließt unwahre Tatsachenbehauptungen nicht kategorisch vom Schutz des Art. 5 GG aus, sondern differenziert teils nach den objektiven, teils nach den subjektiven Umständen; instruktiv dazu etwa BayObLG NJW 1995, 2501, $2502 \mathrm{f}$., das selbst eine Lösung auf der Ebene der Abwägung sucht.

Ständige Rechtsprechung seit BVerfGE $61,1,8$, wo allerdings einschränkend angeführt wird, daß erwiesen oder bewußt unwahre Tatsachenbehauptungen nicht zur Meinungsbildung beitragen. Gegen diese Beschränkung Köbler, NJW 1985, 2389, 2390; Wendt, in: von Münch/Kunig (Anm. 57), Art. 5 Rdn. 10.

59 Tröndle/Fischer, $\ 193$ Rdn. 3; vgl. BGHSt. 12, 287, 293; 18, 182, 184; BGH NStZ 1995, 78; dagegen schließt Lenckner, in: Schönke/Schröder, \193 Rdn. 2, \187 StGB aus.

60 Vgl. Findeisen/Hoepner/Zünkler, ZRP 1991, 245.

61 Zur Unbestimmtheit und grundsätzlichen Zweifeln an der Bestimmbarkeit oben I.

62 Schon oftmals wurde auf die konservierende Wirkung strafrechtlichen Ehrenschutzes hingewiesen, der tradierte gesellschaftliche Achtungsansprüche stabilisiert und damit 
Das letzte Gesellschaftssystem, das versucht hat, sich auf eine überkommene Ordnung zu stützen, da es nicht sicher sein konnte, seine Legitimation jederzeit aufs neue im offenen Streit der Meinungen zu erringen, ist aber vor mehr als zehn Jahren aus Deutschland verschwunden.

Ausgangspunkt der - durch die Untätigkeit des Gesetzgebers mitverursachten - verfassungsrechtlichen Korrekturen am strafrechtlichen Ehrenschutz war die Lüth-Entscheidung ${ }^{63}$, in der das Bundesverfassungsgericht dem Grundrecht nach Art. 5 Abs. 1 Satz 1 GG fundamentale Bedeutung für unser Gesellschaftssystem zumaß und es über seine reine Abwehrfunktion hinaus in eine objektivrechtliche Dimension führte ${ }^{64}$. Die Freiheit der Meinungsäußerung wird seither nicht mehr nur als Grundkonstitution freier Menschen geschützt, sondern auch und vor allem als Möglichkeit der Bürger, an der geistigen Auseinandersetzung im demokratischen System des Grundgesetzes teilzunehmen ${ }^{65}$, das die unmittelbare Teilhabe an politischen Entscheidungen auf die Stimmabgabe bei Wahlen und das Einbringen von Petitionen reduziert. Das Freiheitsrecht wird somit demokratisch instrumentalisiert, um eine möglichst breite Teilnahme am Willensbildungs- und Entscheidungsprozeß zu gewährleisten und den geistigen Boden der politischen Entscheidungen in pluralistischer Weise zu bereiten. Zugleich stellt ein möglichst unverfälschter Wettbewerb der Meinungen sicher, daß gesellschaftliche Entwicklungen und die Notwendigkeit von Änderungen frühzeitig aufgezeigt werden ${ }^{66}$.

Schon diese Erwägungen machen deutlich, daß der strafrechtliche Ehrenschutz in der modernen Grundrechtsordnung, die die Meinungsfreiheit als „die Voraussetzung einer demokratischen Staatsordnung “67, als ein für das

die Arrivierten gegenüber den Außenseitern privilegiert; so namentlich Kübler, JZ 1984, 541, 543f.; ders., NJW 1999, 1281, 1283; auch schon E. A. Wolff, ZStW 81 (1969), S. 886, 894 f.; im Ausgangspunkt z.ustimmend Stürner, JZ 1994, 865, 866. Demgegenüber dient die Meinungsfreiheit gerade der Minderheit als Mittel, die überkommene Ord̉nung zu hintertragen.

63 BVerfGE 7, 198.

64 Zur grundlegenden Bedeutung der Meinungsfreiheit schon BVerfGE 5, 85, 205; zum ganzen auch Meurer, Festschrift für Hirsch, S. 651.

So BVerfGE 5, 85, 205.

66 Zur Fähigkeit des Meinungswettbewerbs, Wechselwirkungen zwischen Handeln und Denken, Regel- und Handelnsordnung zu befördern, aus ökonomischer Sicht Hoppmann, Wirtschaftsordnung und Wettbewerb, 1988, S. $376 \mathrm{ff}$.; eingehend auch Eser, Wahrnehmung berechtigter Interessen als allgemeiner Rechtfertigungsgrund, 1969, S. $51 \mathrm{ff}$, zur Bedeutung des evolutiven Moments in einer wertoffenen Gesellschaft.

67 Grimm, NJW 1995, 1697. 
Staatswesen schlechthin „konstituierendes Element“ 68 betrachtet, einen Fremdkörper darstellt. Um der funktionell-objektiven Bedeutung der Meinungsfreiheit Rechnung zu tragen, hat das Bundesverfassungsgericht das Postulat aufgestellt, bei der Auslegung einer die Meinungsfreiheit begrenzenden Norm sei das Schrankengesetz selbst stets im Lichte der Bedeutung dieses Grundrechts zu interpretieren ${ }^{69}$. Des weiteren hat das Gericht aus der Verfassung einen Satz deduziert, der in seiner die Meinungsfreiheit begünstigenden Dimension Gegenstand vielfacher Kritik ${ }^{70}$, aber andererseits auch selbst Ausgangspunkt einer zweifelhaften Beschneidung der Meinungsfreiheit wurde: Aus dem besonderen Wertgehalt der Meinungsfreiheit folge eine grundsätzliche Vermutung für die Freiheit der Rede in allen Bereichen, namentlich im öffentlichen Leben ${ }^{71}$.

Die Kritik an dieser angeblich den Ehrenschutz relativierenden Rechtsprechung wurde in der Fachwelt um so aufgeregter und polemischer, je stärker Öffentlichkeit und Politik über den Inbalt der zur Entscheidung stehenden Sachverhalte diskutierten ${ }^{72}$. Darüber trat der verfassungs- und strafrechtliche Grundsatzdiskurs in den Hintergrund, so daß die Mitte der neunziger Jahre geführte Kontroverse die Dogmatik des Ehrenschutzes nicht über den status quo ante hinausführte. Dabei wurde nur zu oft verkannt, daß das Bundesverfassungsgericht den Ehrenschutz keineswegs durch die Vornahme einer Einzelfallabwägung generell relativieren oder gar ersetzen wollte ${ }^{73}$, sondern daß es lediglich zu verhindern suchte, daß die Debatte kontroverser und komplexer Themen aus Furcht vor einer Bestra-

$\mathrm{Vgl}$.

Glaeser, JZ 1983, 95, 98; Zuck, JZ 1996, 364, 365, der hervorhebt, das verfassungsrechtliche System basiere auf der Menschenwürde und der freien Entfaltung der Persönlichkeit unter gleichen Bedingungen. Jedoch stellt nicht jede Ehrverletzung eine Verletzung der Menschenwürde dar.

BVerfGE 7, 198, 208. Ähnlich Eser (Anm. 66), S. 45ff., der individualistisch-transsoziale Rechtsgüter von solchen mit immanenten, aus dem Sozialbezug resultierenden Wertkollisionen unterscheidet und für letztere die Frage aufwirft, ob nicht im Zweifel für den Handelnden zu entscheiden sei.

72 Etwa Sendler, ZRP 1994, 343; Brammsen, in: de Boor/Meurer (Hrsg.), Über den Zeitgeist, Bd. II, 1995, S. 303, bei dem besonders deutlich hervortritt, wie schwer es den Kritikern des Bundesverfassungsgerichts fällt, das inhaltsneutrale Spannungsfeld von Kommunikationsfreiheit und Persönlichkeitsrecht ohne Rückgriff auf die (politische) 
fung vereinfachender, aber wirksamer Kritik erstarrt. Keineswegs läßt sich der Rechtsprechung aber ein allgemeiner, irrevisibler Vorrang der Meinungsfreiheit gegenüber dem Ehrenschutz entnehmen ${ }^{74}$; vielmehr sind die Wechselwirkungslehre und die Vermutungsformel ein verfassungsrechtliches $\mathrm{Zu}$ geständnis im - zugegebenermaßen schwierig zu realisierenden - „trade-off“ zwischen Ehrenschutz und ergebnisoffenem Meinungskampf. Daß dieser Ansatz so unvertretbar nicht sein kann, belegt die Tatsache, daß auch der Europäische Gerichtshof für Menschenrechte in seiner Rechtsprechung zu Art. 10 EMRK den grundsätzlichen Vorrang der freien politischen Auseinandersetzung gegenüber dem Ehrenschutz hervorgehoben und sich inhaltlich der Vermutungsregel des Bundesverfassungsgerichts angeschlossen hat $^{75}$.

Die aus normhierarchischen Gründen zwingende Inkorporierung verfassungsrechtlicher Spannungsfelder hat die Ehrschutzdelikte zwar (mehr schlecht als recht) in die nachkonstitutionelle Rechtsordnung gerettet, belastet aber die Dogmatik der Ehrschutzdelikte mit einer zunehmend um die Gegebenheiten des Einzelfalls kreisenden Normanwendung. Die zu einer Schwächung der Rechtssicherheit führenden praktischen Schwierigkeiten sind ihrerseits aber nur ein Reflex der pluralistischen und spannungsgeladenen Wertordnung des Grundgesetzes, die auf das strafrechtliche Rechtsgut der Ehre ausstrahlt ${ }^{76}$. Die verfassungsrechtlichen Interessenkonflikte sind schon für die Bestimmung des Rechtsguts von Bedeutung. Man kann ihnen nicht mit einem pauschalen Hinweis auf $₫ 1.93$ StGB Rechnung tragen ${ }^{77}$, zumal diese Vorschrift objektiv und subjektiv durch das Kriterium der „eigenen berechtigten“ Interessen begrenzt ist, während Art. 5 GG auch die Wahrnehmung fremder und nur subjektiv berechtigt erscheinender Interessen schützt und fördert. Die verfassungsrechtlichen Spannungen wirken sich also nicht erst auf die Frage nach einer etwaigen Rechtfertigung aus, sondern zwingen dazu, das von den $\mathbb{\$} 185 \mathrm{ff}$. StGB geschützte Rechtsgut insgesamt in den Blick nehmen.

74 Hingegen gesteht der U.S. Supreme Court der Meinungsfreiheit im öffentlichen Diskurs auch in Extremfällen generellen Vorrang zu, da man annimmt, eine Gesellschaft mit einem offenen Meinungsklima generiere bessere Ergebnisse auch für den einzelnen als eine staatlich kontrollierte Kommunikation; vgl. dazu Kübler, AöR 2000, 109. EGMR NJW 1999, 1321.

76 Dazu Müller-Dietz, Gedächtnisschrift für Zipf, 1999, S. 123 f.; zur Unbestimmtheit des strafrechtlichen Rechtsgutes oben I.

77 So aber wohl Meurer, Festschrift für Hirsch, S. 651, $656 \mathrm{ff}$. 
Als schwerwiegend erweist sich im übrigen auch eine anfangs wohl ungeplante Nebenwirkung der - durchaus wohlmeinenden - Rechtsprechung des Bundesverfassungsgerichts, deren Tragweite im Schlachtenlärm früherer Kontroversen unterzugehen drohte: die Differenzierung des Schutzumfangs von Art. 5 Abs. 1 GG und damit letztlich auch der Strafbarkeit nach dem Inhalt der Äußerung und dem Kommunikationsumfeld. Nach Auffassung der Rechtsprechung ist zwischen Meinungsäußerungen zu den die Öffentlichkeit wesentlich berührenden Fragen einerseits und Äußerungen im privaten Bereich andererseits zu unterscheiden, wobei letztere oftmals mit pauschalen Verdikten (sie seien "ohne allgemeines Interesse“ ${ }^{78}$ ) desavouiert und per se für weniger schutzwürdig erachtet werden als Stellungnahmen zu bereits publikumswirksam gewordenen Themen ${ }^{79}$. Hier drängt sich die Frage auf, welche gesellschaftliche Instanz sich zu entscheiden anmaßen will, ob ein Thema von allgemeinem Interesse ist oder die Öffentlichkeit wesentlich berührt ${ }^{80}$. Wegen der konstitutionellen Unwissenheit aller Individuen - also auch der Richter, Parlamentarier und Wissenschaftler - vermag niemand zu sagen, welchen Personen man zu einem bestimmten Zeitpunkt die erforderlichen Freiheitsräume zur Verfügung stellen muß, damit diese auf Fehlentwicklungen hinweisen und Lösungswege aufzeigen können. Kann sich aber niemand ein solches Wissen anmaßen, so wird man allen Akteuren einen gleich großen Freiheitsraum einräumen müssen ${ }^{81}$. Soweit eine breite Diskussion in den Medien als Indiz für ein öffentliches Interesse herangezogen wird, werden Themen, die bereits Zugang zum Meinungsmarkt gefunden haben, gegenüber „NewcomerThemen" privilegiert, die zunächst nur in kleineren Kreisen debattiert werden, oftmals aber das wenig später auf dem Forum der breiten Öffentlichkeit kontrovers Diskutierte antizipieren. Die Funktion privater Meinungsäußerungen in einer demokratischen Gesellschaft ${ }^{82}$ ist denn auch vom Bundses-

79 Ständige Rechtsprechung seit BVerfGE 7, 198, 212; siehe etwa BVerfGE 12, 113, 127; 24, 275, 282f.; 54, 129, 137; 66, 116, 150; 68, 226, 232; 82, 236, 260; zustimmend Meurer, Festschrift für Hirsch, S. 660f.; kritisch etwa Scholz/Konrad, AöR 1998, 60, $102 \mathrm{ff}$; Schmitt Glaeser, JZ 1983, 95, $98 \mathrm{ff}$; sich an dessen Argumentation wortgleich anlehnend Stark (Anm. 9), S. $113 \mathrm{ff}$.

80 Ähnlich Lerche, Festschrift für G. Müller, 1970, S. 197, 213 f., der in der Bewertung der Gemeinschaftswidrigkeit einen grundsätzlich unzulässigen Verstoß gegen das Gebot der inhaltsneutralen Bewertung durch den Staat und seine Untergliederungen sieht.

82 Dazu auch Häberle, Die Verfassung des Pluralismus, 1980, S. $126 \mathrm{ff} ., 140 \mathrm{f}$. 
verfassungsgericht zunächst durchaus erkannt worden ${ }^{83}$; später hat es den Schutzbereich jedoch ohne Begründung auf die politische Debatte reduziert $^{84}$.

Grundsätzlich muß sich das Bundesverfassungsgericht den Einwand gefallen lassen, allzu schematisch politischen Themen ein Allgemeininteresse, privaten Äußerungen hingegen schlichten Eigennutz zu attestieren ${ }^{85}$. Die Erkenntnisse der Konstitutionenökonomik ${ }^{86}$ lehren demgegenüber, daß sich im Gewande öffentlicher Wortmeldungen und unter dem Banner des Gemeinwohls oft handfeste Privilegieninteressen verbergen, so daß eine Unterscheidung zwischen eigennützigen Verhaltensweisen im geschäftlichen oder privaten Verkehr auf der einen Seite und altruistischem, politischem Handeln auf der anderen Seite an den Realitäten vorbeigeht. Fremdund eigennützige Interessen lassen sich mithin im Rahmen von Meinungsäußerungen kaum voneinander trennen. Zudem verkennt die Differenzierung, daß der private wie der politische Bereich von Rationalitätsaxiom und methodologischem Individualismus ${ }^{87}$ geprägt ist und daß die Akteure die Alternativen in beiden Bereichen stets im Hinblick auf "gains from trade“ gegeneinander abwägen ${ }^{88}$. Mit der Bevorzugung des politischen Diskurses diskriminiert das Bundesverfassungsgericht den grundsätzlich ebenso schützenswerten privaten Raum durch ein ungleich strengeres Strafrecht. Auch hier stellt sich also die Gerechtigkeitsfrage ${ }^{89}$. Die verfassungsrechtlich gebotenen "Notoperationen" des Bundesverfassungsgerichts haben also nicht nur zu großer Rechtsunsicherheit bej den Ehrschutzdelikten geführt, sondern de facto auch zu einer Zweiklassen-Rechtsprechung, die die private Kommunikation strafrechtlich diskriminiert.

So hebt BVerfGE 7, 198, 208, hervor, die Vermutung für die Freiheit der Rede gelte „in allen Bereichen".

Ab BVerfGE 12,113,127, findet sich nur noch der insoweit verstümmelte und sinnentstellende Hinweis auf BVerfGE 7, 198, 212. Die dortige Aussage, die Gewährleistung gelte in allen Bereichen, wird ignoriert.

Scholz/Konrad, AöR 1998, 60, 104.

Einführend Vanberg, in: Korff u.a. (Hrsg.), Handbuch der Wirtschaftsethik, Bd. 1, 1999, S. $504 \mathrm{ff}$.

Schumpeter, Das Wesen und der Hauptinhalt der theoretischen Nationalökonomie, 1908, S. $88 \mathrm{ff}$.

Zu diesen Erkenntnissen J. M. Buchanans siehe Vanberg (Anm. 86), S. 505, und Hoppmann (Anm. 66), S. $160 \mathrm{ff}$.

Kiesel, NVwZ 1991, 1129, 1130; Schmitt Glaeser, JZ 1983, 95, 98; Scholz/Konrad, AöR 1998, 60, 105, letztere mit dem Hinweis auf den engen Bezug der privaten Kommunikation zur Menschenwürde. 
Dieser Befund erlaubt die grundsätzliche Prüfung, ob sich verfassungsrechtliche Bedenken gegen eine Abschaffung der Ehrdelikte als die zwar radikal anmutende, im Ergebnis aber gerechtere Auflösung der verfassungsrechtlichen Spannungen erheben. Dabei wird durchaus nicht verkannt, daß auch das Freiheitsrecht des Art. 5 Abs. 1 GG Grenzen, namentlich in bezug auf den Ehrenschutz unterliegt. Die Anerkennung des Verfassungsrechtsguts „Ehre“ entzieht jedoch der Kritik am strafrechtlichen Schutz dieses Rechtsguts nicht den Boden: Zum einen können Verfassungsgüter weitflächiger definiert werden als Strafrechtsgüter und sind auch vom Postulat des Art. 103 Abs. 2 GG freigestellt. Zum anderen folgt aus der Zuerkennung eines verfassungsrechtlichen Achtungsanspruches nicht etwa ein strafrechtlich zu schützendes „Grundrecht auf Ehre" 90 , sondern nur die Notwendigkeit irgendeiner staatlichen Reaktion auf seine Verletzung ${ }^{91}$.

Da keinem der konfligierenden Rechtsgüter per se eine Vorrangstellung zukommt ${ }^{92}$, muß es der verfassungsinterpretierende Gesetzgeber dabei belassen, äußere Grenzen abzustecken, jenseits derer dem Ehrenschutz Vorrang vor der Meinungsfreiheit zu geben ist. Eine evidente Grenze ergibt sich insoweit nur aus der hervorgehobenen Stellung der Menschenwürdeverbürgung in Art. 1 Abs. 1 GG. Meinungsäußerungen, die einem Grundrechtsträger die allen zustehende Personenwürde aberkennen, können vor der Verfassung keinen Bestand haben. Jedoch sei davor gewarnt, den Kreis der die Menschenwürde tangierenden Ehrverletzungen zu sehr auszudehnen oder gar das allgemeine Persönlichkeitsrecht - trotz der zweifellos vorhandenen Nähe - in den Schutzbereich von Art. 1 Abs. 1 GG zu ziehen ${ }^{93}$. Eine Überschreitung der zulässigen Meinungsfreiheit durch Verletzung der Menschenwürde beschränkt sich auf den Bereich, der unter dem Topos der Schmähkritik ${ }^{94}$

90 Auf diesen Unterschied weist hin Herzog, in: Maunz/Dürig/Herzog/Scholz, GG, Stand 1982, Art. 5 Abs. 1, 2 Rdn. 287.

91 Schmidhäuser, Allg. Teil, 2. Aufl. 1984, S. 84 f.; vgl. auch Müller-Dietz, Gedächtnisschrift für Zipf, S. $123 \mathrm{ff}$.; siehe ferner unten bei Anm. 99 ï.

92 Meurer, Festschrift für Hirsch, S. 653.

93 Bedenklich Peglau, ZRP 1998, 249, 250. Hingegen warnte schon Dürig, in: Maunz/ Dürig/Herzog, GG, Art. 1 Abs. 1 Rdn. 29, vor einer Umwandlung der Menschenwürde in „kleine Münze“, da Art. 1 Abs. 1 GG viel wichtigere Aufgaben wahrzunehmen habe als die Ausdehnung des persőnlichen Ehrenschutzes. Differenzierend auch schon Eser (Anm. 66), S. 48, der die Ehre als ein Gut bezeichnet, das im Gegensatz zur Menschenwürde nicht jenseits der Gemeinschaft stehe, sondern als Autoritätsund Einflußfaktor zugleich auch wieder in den Alltagskampf der Interessen zurückwirke.

94 Dazu Leibholz/Rink/Hesselberger, GG, Stand 1993, Art. 5 Rdn. 992, m. w. N. 
diskutiert wird ${ }^{95}$. Mit einer solchen Beschränkung muß nicht notwendig eine „Verrohung der Sitten“ einhergehen, wie von besorgter Seite befürchtet wird ${ }^{96}$, denn es ist keineswegs wahrscheinlich, daß die Abnehmer von Meinungen sich von rohen, unsachlichen oder gar verletzenden Darstellungen einnehmen und überzeugen lassen; im Gegenteil wird in nicht wenigen Fällen der zu solchen Mitteln Greifende selbst eher auf Ablehnung stoßen oder sogar Mitleid und Solidarität zugunsten des Gescholtenen hervorrufen. Solche sozialen Reaktionen werden auch den „Meinungsproduzenten“ nicht verborgen bleiben, die dann schon aus rationalen Erwägungen ihr Verhalten modifizieren werden ${ }^{97}$. Auf diese Weise tragen die am Kommunikationsprozeß Beteiligten zu einer schnelleren und vor allem genaueren Anpassung der Darstellungsweise an die sich dynamisch entwickelnden Anschauungen der Gesellschaft bei, als Sanktionen staatlicher Instanzen dies jemals könnten.

Freilich wird sich nicht jeder, der sich ungerechtfertigt angegriffen oder in die Arena der Öffentlichkeit gezerrt sieht, mit der Aussicht trösten lassen, als „Bewertungsparameter“ gedient und als Opfer zu einem Anschauungswandel beigetragen zu haben. Zwar wird man „public figures“ zumuten können, den Druck der öffentlichen Meinung auszuhalten, dem sie sich freiwillig ausgesetzt haben und den sie im allgemeinen auch durchaus zu tragen bereit sind ${ }^{98}$. Aber auch in bezug auf andere Personen muß man nicht unbedingt nach dem Staatsanwalt rufen, wenn man eine Beschränkung der Meinungsfreiheit und den Schutz der Ehre für notwendig hält. Insbesondere vor dem Hintergrund der „ultima ratio“-Funktion des Strafrechts kann vielmehr auch der zivil- und medienrechtliche Weg eine hinreichende Möglichkeit der „Ehrenrettung“ sein.

Eine Verpflichtung zum Erlaß strafrechtlicher Normen hat das Bundesverfassungsgericht bislang nur in einem Ausnahmefall - für den Schutz des ungeborenen Lebens - aus dem Grundgesetz deduziert ${ }^{99}$. In diesem Urteil hat das Gericht überdies mit Recht hohe Hürden für die Annahme ver-

as Kübier, AöR 2000, 109, 125, will zudem Art. 5 Abs. 1 GG beschränken, wenn die Äußerungen zur Stigmatisierung und sozialen Ausgrenzung bestimmter Personen verwendet werden.

96 So etwa Stark (Anm. 9), S. 115.

97 Es sei an dieser Stelle nur darauf hingewiesen, daß jedenfalls in der amerikanischen Gesellschaft der Verzicht auf Strafrecht einen grundlegenden Wandel der puritanischen Sittenvorstellungen nicht herbeigeführt hat.

98 Die Ansicht, ein schneidiger strafrechtlicher Ehrenschutz würde den Eintritt auch sensiblerer Menschen in die Politik befördern, dürfte in den Ohren von Berufs- und Ehrenamtspolitikern grotesk klingen.

BVerfGE 39, 1, 45. 
fassungsrechtlicher Bestrafungspflichten aufgestellt, verkehren diese doch den Ausgangspunkt der Entwicklung der Grundrechte - als Abwehrrechte gegen den Staat - geradezu in sein Gegenteil ${ }^{100}$. Selbst wenn man annimmt, $\mathrm{da} ß$ die in den Abtreibungs-Entscheidungen aufgestellten Kriterien allgemein gültig seien ${ }^{101}$, verdeutlicht ein Blick auf die nach Ansicht des Gerichts eine Pönalisierungspflicht begründenden Topoi, daß einer Entkriminalisierung der Ehrdelikte keine verfassungsrechtlichen Hindernisse entgegenstehen würden. Zunächst ist schon das Rechtsgut „Ehre“ von einer solchen Unschärfe und subjektiv geprägten Bedeutung, daß einem Pönalisierungsgebot die Schwierigkeit einer hinreichend bestimmten Fassung des Verbots entgegensteht. Auch in bezug auf Äußerungen, die den Kernbereich des allgemeinen Persönlichkeitsrechts tangieren, läßt sich mit Hinweis auf die Berührung mit der Menschenwürde keine verfassungsrechtliche Verpflichtung zum Strafen begründen ${ }^{102}$, denn selbst wenn es gelänge, einen solchen Kernbereich eindeutig zu konturieren ${ }^{103}$, würde die Umsetzung in einen justiziablen Straftatbestand auf zumindest ebenso große Probleme stoßen, wie sie bereits heute die Ehrdelikte ihren Interpreten bereiten. Entscheidend gegen eine Pönalisierungspflicht spricht aber der „verfassungsrechtliche Kurzschluß "104, dem diejenigen unterliegen, die aus einem durch das Grundgesetz begründeten Schutzgebot - gleich welchen Rechtsguts - ein Strafbarkeitsgebot folgern ${ }^{105}$. Dabei verlangt schon das Verhältnismäßigkeitsprinzip die Prüfung, ob der Einsatz des Strafrechts zum Schutz des Rechtsguts geeignet ist, ohne gleichzeitig die Freiheitskreise anderer unnötig zu beschränken ${ }^{106}$. Die an der Kriminalstatistik abzulesenden Unzuläng-

100 Im Sondervotum (BVerfGE 39, 1, 73) wird ausgeführt, eine aus den Grundrechten abgeleitete Pflicht zum Strafen bedeute, daß die „Grundrechte unter der Hand aus einem Hort der Freiheitssicherung zur Grundlage einer Fülle von freiheitsbeschränkenden Reglementierungen werden“.

101 Insoweit zweifelnd Müller-Dietz, Festschrift für Dreher, 1977, S. 97, 114, der die Argumentation des Bundesverfassungsgerichts als „offenkundig zugeschnitten auf den Schutz elementarer Rechtsgüter und Grundwerte" ansicht; auch Eser (Anm. 66), S. 47, weist insoweit auf die herausgehobene Steilung des Rechtsguts Leben hin.

102 So aber Peglau, Der Schutz des allgemeinen Persönlichkeitsrechts durch das Strafrecht, 1997, S. $47 \mathrm{ff}$.

103 Wenn selbst hinsichtlich des Einflusses der Menschenwürde auf das Rechtsgut „Leben“ Unsicherheiten bestehen (vgl. Maunz/Zippelius, Deutsches Staatsrecht, 29. Aufl. 1994, S. 167), so gilt dies erst recht für das allgemeine Persönlichkeitsrecht.

104 Herzog, JR 1969, 441, 445.

105 Insoweit kurzschlüssig Peglau (Anm. 102); ders., ZRP 1998, 249, 250.

106 Auch BVerfGE 39, 1, 45, berücksichtigt sowohl die praktische Wirksamkeit als auch die Substituierbarkeit durch andere rechtliche Mittel; vgl. auch Müller-Dietz (Anm. 91), S. 108f. 
lichkeiten der Ehrschutzdelikte und die Möglichkeit des Ausweichens auf zivil- und medienrechtliche Rechtsbehelfe sprechen, wenn nicht schon gegen die Geeignetheit, so doch gegen die Erforderlichkeit dieser Normen. Wer dennoch die Notwendigkeit strafrechtlicher Sanktionen unmittelbar aus der Verfassung ableiten will, muß sich den Vorwurf gefallen lassen, einem Staatsverständnis von Befehl und $Z$ wang den Vorzug gegenüber differenzierteren Mitteln einzuräumen, die dem Staat zur Flankierung privaten Rechtsschutzes zur Verfügung stehen ${ }^{107}$. Der staatlichen Intervention in das Verhältnis der Staatsbürger untereinander bedürfte es nur, wenn strafrechtliche Sanktionen gegen Meinungsäußerungen eine sozialpädagogische Wirkung oder gar ein Mittel zur „Bewußtseinsprägung" versprächen ${ }^{108}$. Wenn aber das Strafgesetzbuch überhaupt zum „Volkslehrbuch des ethischen Minimums " 109 funktionalisiert werden soll, stellt sich die Frage, ob von den evident nicht funktionstüchtigen Mitteln des Ehrenschutzes nicht sogar gesellschaftspolitisch kontraproduktive Signale ausgehen ${ }^{110}$.

Die verfassungsrechtlich begründete Argumentation drängt also - im Gegensatz zu bloß intuitiven Vorbehalten - zu der Schlußfolgerung, daß man die Verantwortung für einen funktionierenden Kommunikationsprozeß auf die Bürger übertragen sollte, aus deren Mitte ethische und soziale Verhaltensnormen entstehen, statt den strafrechtlichen Ehrenschutz samt den mit ihm verbundenen erheblichen Beschneidungen der Meinungsfreiheit beizubehalten

\section{IV. Äußerungsdelikte in ihrer transnationalen Dimension}

Es sind nicht nur die aus der deutschen Rechtsordnung resultierenden Bedenken, die für eine Entkriminalisierung des Ehrenschutzes streiten, sondern zunehmend auch transnationale Ent- und Verwicklungen, die sich aus der Allgegenwärtigkeit des Internet und der durch dieses Medium verbreiteten Informationen und Meinungen für das Strafrecht ergeben. Wie für eine Vielzahl von Tatbeständen des Besonderen Teils, so lassen sich auch und

107 Herzog, JR 1969, 441, 445.

108 So Peglau, ZRP 1998, 249, 250, der die strafrechtlichen und kriminalpolitischen Probleme des Ehrenschutzes nicht hinreichend würdigt.

109 Kriele, JZ 1975, 222.

110 Zur Beachtlichkeit „schädlicher Nebenwirkungen“ des Einsatzes strafrechtlicher Sanktionen Müller-Dietz (Anm. 91), S. 109. 
gerade für die Ehrschutzdelikte zwei Problemkreise konturieren. Zum einen geht es um die Frage, an welcher Strafrechtsordnung ein Internetnutzer seine Meinungsäußerungen im weltweiten Datenverkehr auszurichten hat, und zum anderen um das rechtspolitische Problem, ob die im Internet angelegte Regelmäßigkeit der Grenzüberschreitung nicht zwangsläufig zu einer Anpassung des materiellen Strafrechts zumindest in den Bereichen führen muß, für die sich die Benutzung von internationalen Computernetzen geradezu aufdrängt.

Will man das Internet nicht insgesamt der deutschen Strafgewalt unterwerfen und damit sämtliche Teilnehmer aus den 150 an das Internet angeschlossenen Staaten zu Adressaten der $\$ \$ 185 \mathrm{ff}$. StGB machen, dann wird man von Auffassungen Abschied nehmen müssen, die mit unterschiedlicher Begründung bei Äußerungsdelikten stets einen Erfolgseintritt im Inland und, insoweit dann zwingend, nach $\mathbb{\$}$ 3, 9 Abs. 1 Var. 3 StGB die deutsche Strafgewalt bejahen ${ }^{111}$. Daraus ergäben sich nicht nur unübersehbare praktische Schwierigkeiten für die durch $\ 152$ Abs. 2 StPO gebundenen Strafverfolgungsbehörden; die völkerrechtlich problematische Annahme deutscher Jurisdiktion in bezug auf Internetnutzer und Provider ${ }^{112}$ würde auch die verhaltenssteuernde Wirkung des Strafrechts verfehlen, da von deutschen Behörden nicht auf ausländische Server eingewirkt werden kann ${ }^{113}$. Bei der Einordnung von Delikten, die im Internet begangen werden, in das tradierte System des „transnationalen Strafrechts" 114 ist somit grundsätzlich ein behutsames Vorgehen angezeigt, das der dynamischen Entwicklung nicht vorgreift oder sie gar behindert ${ }^{115}$.

Wenn auf der Grundlage von $₫ 7 \mathrm{StGB}$ die Anwendung der deutschen Beleidigungstatbestände auf Daten bejaht wird, die mittels des Internet

111 Conradi/Schlömer, NStZ 1996, 366, 368; Kuner, CR 1996, 453, 455 f.; Sieber, JZ 1996, 429, 430; bedenklich weit auch BT-Drucks. 13/4800, S.8; neuerdings auch BGH NJW 2001, 624, 627f. Kritisch zu diesen. Tendenzen auch schon Eser, in: Roxin/Widmaier (Hrsg.), 50 Jahre Bundesgerichtshof - Festgabe aus der Wissenschaft, Bd. IV, 2000 , S. $1,20 \mathrm{ff}$.

112 Zur Mitverantwortlichkeit des Providers für strafrechtlich relevante Inhalte Sieber, in: Hoeren/Sieber, Handbuch für Multimedia-Recht, 2000, Rdn. $219 \mathrm{ff}$. zu Teil 19.

113 Sieber (Anm. 112), Rdn. 411 zu Teil 19.

114 Zur Überlegenheit dieses Begriffes Eser, in: Schönke/Schröder, vor \$S 3-7 Rdn. 2.

115 Als Begehungsort von Beleidigungsdelikten kommt neben dem Aufenthaltsort des Täters auch der Standort des Servers in Betracht, auf dem die beleidigenden Inhalte zum Abruf bereit gestellt werden; so Cornils, JZ 1999, 394, 397; Eser, in: Schönke/ Schröder, $\$ 9$ Rdn. 4 . 
nach Deutschland transportiert werden, so wird zumeist davon ausgegangen, daß alle relevanten Rechtskreise ähnliche Strafvorschriften enthalten ${ }^{116}$. Doch schon der Blick in das deutschsprachige Ausland widerlegt diese These, stellt doch zum Beispiel das österreichische Strafgesetzbuch die einfache, nicht öffentliche Beleidigung nicht unter Kriminalstrafe ( $\mathbb{} 115$ ö.StGB). Für die Begründung der Strafgewalt nach $₫ 7 \mathrm{Abs.} 1$ oder $2 \mathrm{StGB}$ resultieren daraus Probleme bei der Feststellung einer Tatortstrafbarkeit. Sehr zu bezweifeln ist auch, ob es gelingen würde, für eine vor einem deutschen Gericht zur Anklage gebrachten Beleidigung wegen Leugnen des Holocaust ${ }^{117}$ eine hinreichende amerikanische Strafbestimmung nachzuweisen, wird doch schon die einfache Beleidigung in den USA von den Strafverfolgungsbehörden jedenfalls de facto nicht mehr verfolgt ${ }^{118}$, während die „Auschwitzlüge" vollends eine deutsche Besonderheit ohne Entsprechung ist ${ }^{119}$. Will man den unterschiedlichen rechtsethischen Vorstellungen potentieller Täter eines Beleidigungsdelikts nicht erst im Rahmen eines Verbotsirrtums Rechnung tragen ${ }^{120}$ oder wegen des Bagatellcharakters eine Verfahrenseinstellung befürworten ${ }^{121}$, so lassen sich die Ressourcen der deutschen Strafverfolgungsorgane wohl nur dann schonen, wenn die deutsche Jurisdiktion für Fälle dieser Art von vornherein verneint wird. Auf der anderen Seite besteht dann aber das Problem einer Inländerdiskriminierung insofern, als Beleidigungen im Inland stets strafrechtlich geahndet werden, während sich derjenige vor Strafbarkeit sicher wissen kann, der mittels des Internet aus einem Land operiert, das der Meinungsfreiheit größeres Gewicht beimißt und Aussagen wie z. B. das Leugnen des Holocaust nicht mit Strafe bedroht. Gegen solche Aussagen erhebt sich völlig zu Recht das Pietätgefühl; sie sollten aber im wesentlichen als das behandelt werden, was sie sind: tumber Unfug.

116 So auch Ringel, CR 1997, 302, 307.

117 BGHZ 75, 160, 163 ff.; BGHSt. 40, 97, 103 f.; kritisch Lenckner, in: Schönke/Schröder, $\{185 \mathrm{Rdn} .3$.

118 Timm, Schutz von Amtsträgern vor diffamierenden Äußerungen in der Presse in Deutschland und in den USA, 1999, S. 172; Phelbs/Hamilton, Libel: Rights, Risks, Responsibilities, 1966, S. 360.

119 Zur Rechtsprechung des U.S. Supreme Court jüngst Kübler, AöR 2000, 108, $117 \mathrm{f}$.

120 So aber wohl Hilgendorf, NJW 1997, 1873, 1876. Zur Abgrenzung eines Verbotsirrtums von einer Fehlvorstellung über die Anwendbarkeit deutschen Strafrechts Eser (Anm. 66), S. $17 \mathrm{ff}$.

121 Bär, in: Rossnagel (Hrsg.), Recht der Multimediadienste, 2000, Rdn. 45f. zu Teil 7, fordert die Beachtung der Grundsätze über die Verfahrenseinstellung für Bagatelldelikte auch bei Beleidigungen im Internet. 
Somit stellen die aus dem Internet auf deutsche Regulierungsinseln zulaufenden Liberalisierungswellen nicht nur wettbewerbsrechtliche Eigenheiten wie das Rabattgesetz und die Zugabeverordnung, sondern auch solche Delikte in Frage, deren strafrechtlicher Schutz nicht zum Gemeingut aller zivilisierten Nationen gehört, wie namentlich die Ehrschutzdelikte.

\section{V. Überlegenheit außerstrafrechtlichen Ehrenschutzes}

Bereits die im grundsätzlichen liegende Mangelhaftigkeit strafrechtlichen Ehrenschutzes spricht für dessen Abschaffung und belastet die denkbare Alternative einer möglichen Reform der Tatbestände mit erheblichen Zweifeln ${ }^{122}$. Es wird dabei freilich nicht übersehen, daß sich auch das Zivilrecht als nächstliegende Alternative zum strafrechtlichen Ehrenschutz den Aufgaben einer hinreichenden Konturierung des Rechtsguts und dessen Abwägung gegenüber der Meinungsfreiheit stellen muß. Gefahr droht dem freien Kommunikationsprozeß auch durch einen ausufernden zivilrechtlichen Persönlichkeitsschutz, namentlich die zensorische Wirkung eines unverhältnismäßigen und übertriebenen (Straf-)Schadensersatzes darf nicht unterschätzt werden ${ }^{123}$. Gleichwohl ist das Zivilrecht nicht nur wegen der größeren Freiheit in der Tatbestandsbeschreibung eher als das Strafrecht für Abwägungen und Wertungen empfänglich, treten sich die Kontrahenten doch auf der Ebene der Gleichordnung gegenüber und verbindet sich mit einer zivilgerichtlichen Entscheidung über die $\ddot{A} u ß e r u n g$ nicht zwangsläufig auch der sozialethische Tadel über den $\ddot{A} u ß e r n d e n$, wie er in einer strafrechtlichen Verurteilung zum Ausdruck kommt ${ }^{124}$.

Der deliktsrechtliche Anspruch des Verletzten war zwar ursprünglich über $₫ 823$ Abs. 2 BGB untrennbar mit den Beleidigungstatbeständen des

122 Anders Hirsch, Festschrift für Wolff, S. 125, 126f;; Otto, Festschrift für Schwinge, S. 71, 73.

${ }^{123}$ Eine Auflösung des Spannungsverhältnisses zwisc'nen E'nrenschutz und Mieiriungsfreiheit ist ebensowenig das Anliegen dieser Abhandlung wie die Erfassung sämtlicher vom Persönlichkeitsrecht umfaßter Interessen des einzelnen. Vielmehr soll hier das grundsätzliche Problem des richtigen Ortes und der angemessenen Mittel zur Lösung dieser Fragen behandelt werden.

124 Gerade darin sieht freilich Hirsch, Festschrift für Lange, S. 815, 822, eine Schwäche des zivilrechtlichen Ehrenschutzes; er verweist darauf, daß es insbesondere dem Opfer darum gehe, den Verletzer als verantwortlichen Täter zur Rechenschaft zu ziehen. Ob eine solche Erwartung jedoch Strafe oder Buße rechtfertigt, darf nicht zuletzt vor dem Hintergrund der ultima ratio staatlicher Sanktion bezweifelt werden; vgl. dazu bereits oben II. (a. E.). 
Strafgesetzbuchs verbunden, jedoch genießt die Ehre unter dem bestimmenden Einfluß des Grundgesetzes längst den Schutz nach $₫ 823$ Abs. 1 BGB. Als besonderes unter den allgemeinen Persönlichkeitsrechten, die der Bundesgerichtshof und das Bundesverfassungsgericht aus Art. 2 Abs. 1 GG i.V.m. Art. 1 Abs. 1 GG abgeleitet haben ${ }^{125}$, hat die Ehre entgegen den Vorstellungen des - verfassungsrechtlich insoweit noch ungebundenen - Gesetzgebers als sonstiges Recht Eingang in $\ 823$ Abs. 1 BGB gefunden ${ }^{126}$. Der Schutz der Ehre durch das Zivilrecht hat sich so mit Hilfe des Verfassungsrechts vom Strafrecht nicht nur emanzipiert ${ }^{127}$, sondern zeigt seine Überlegenheit bereits de lege lata in der breiteren Erfassung relevanter Sachverhalte, den flexibleren Rechtsfolgen und dem stärker selbstbestimmten Verfahren. De lege ferenda verdienen die Ausweitung einer Geldentschädigung bei schwerwiegenden Persönlichkeitsverletzungen und insbesondere ein in seinen Voraussetzungen weniger restriktiver Anspruch auf Gegendarstellung Beachtung, mit dem sich Waffengleichheit auf dem Forum der Öffentlichkeit erreichen läßt.

Von der Fessel des Art. 103 Abs. 2 GG befreit ${ }^{128}$, bedient sich das Zivilrecht zum Schutz der Ehre der Figur des allgemeinen Persönlichkeitsrechts. Dieser Begriff vermag jenseits zulässiger strafrechtlicher Ehrdefinitionen Herabsetzungen und Kränkungen ebenso aufzunehmen wie das Recht des einzelnen auf informationelle Selbstbestimmung und den Schutz vor kommerzieller Ausnutzung der Person ${ }^{129}$. Auch für juristische Personen und Kollektive läßt sich ein ihrem Wesen und ihren zugewiesenen Funktionen angemessener Persönlichkeitsschutz leichter begründen ${ }^{130}$, ohne in die Irrationalität einer fiktiven Kollektivehre fliehen zu müssen ${ }^{131}$. Die untaug-

125 Grundlegend BGHZ 13, 334, 338; BVerfGE 34, 269; vgl. zur weiteren Entwicklung nur Larenz/Canaris, Schuldrecht Bes Teil II/2, 13. Aufl. 1994, S. 491 ff.; Schwerdtner, in: MüKO BGB, 3. Aufl. 1993, \12 Rdn. 186ff.; Seifert, NJW 1999, 1889, 1891 ff., jeweils m.w. N.; zur aktuellen Entwicklung der Rechtsprechung Soebring/Seelmann-Eggebert, NJW 2000, 2466.

126 V gi. nur Hager, AcP 196, i68, 172.

127 Vgl. zum Einfluß des Verfassungsrechts auf den zivilrechtlichen Ehrenschutz Seyfabrt, NJW 1999, 1287.

128 Es sei an dieser Stelle nur darauf hingewiesen, daß eine Überfrachtung des Privatrechts mit strafrechtlichen Elementen eine Anwendung von Art. 103 Abs. 2 GG auch auf das Privatrecht nahelegen könnte; vgl. Gounalakis/Rösler (Anm. 45), S. 97.

129 Arzt, JuS 1982, 717, 725; Larenz/Canaris (Anm. 125), S. 517; Kübler, NJW 1999, 1281, $1282 \mathrm{f}$.

130 BGHZ 81, 75, 78; Larenz/Canaris (Anm. 125), S. 520.

131 Daher sollte auch die Zivilrechtsprechung auf den „Ehrenschutz" - so ausdrücklich BGHZ 78, 24, 25; 98, 94, 97; BGH NJW 1975, 1882, 1884; NJW 1981, 2117, 2119 - 
lichen Versuche, strafrechtliche Begriffe zu bilden, die zugleich exakt und leistungsfähig sind, können daher zugunsten einer der Person insgesamt gerecht werdenden Erfassung ihrer schutzwürdigen Interessen aufgegeben werden.

Ein weiterer Vorteil zivilrechtlichen Ehrenschutzes zeigt sich darin, daß die Reaktionen der Rechtsordnung auf eine Aussage nicht notwendig an deren Inhalt oder - insoweit bedingt - an deren Wahrheit anknüpfen müssen ${ }^{132}$. Das Urteil kann den Teilnehmern am Kommunikationsprozeß überlassen bleiben, sobald der gleiche Zugang zu den Kommunikationsmitteln gewährleistet wird, wenn also Angegriffener und Äußernder gleichermaßen in der Lage sind, sich Gehör zu verschaffen ${ }^{133}$. Verwehren Meinungs- und Kommunikationskartelle dem einzelnen diesen Zugang, so liegt bereits darin - unabhängig vom Gehalt der Äußerungen - eine Verletzung des allgemeinen Persönlichkeitsrechts, die eine Reaktion der Rechtsordnung erfordert. Die Straftatbestände der Beleidigung mit ihrem am Inhalt ausgerichteten Ehrverständnis können diese Anknüpfung dagegen nicht leisten und erweisen sich, indem sie mit dem Anspruch auf Verbindlichkeit inhaltliche Maßstäbe zugrundelegen, jedenfalls bei Werturteilen als vordemokratisch ${ }^{134}$, unzeitgemäß und gegenläufig zur internationalen Entwicklung ${ }^{135}$.

Die Unterlegenheit strafrechtlichen Ehrenschutzes setzt sich bei den Rechtsfolgen fort. Die Kriminalstrafe kommt regelmäßig nicht nur zu spät, sie kann auch nicht angemessen variiert werden, und der sie tragende Strafzweck der Prävention wird durch das tatsächliche Verfahren entwertet ${ }^{136}$. Anders dagegen die außerstrafrechtlichen Maßnahmen: Ansprüche auf Gegendarstellung, Widerruf, Unterlassung und Schadensersatz stehen in einem sich ergänzenden Verhältnis zueinander und erlauben dem Verletzten in ihrer alternativen oder kumulativen Anwendung eine seinen Interessen und der Schwere des Eingriffs angemessene Reaktion ${ }^{137}$. Die Zivilgerichte gewährleisten deren Durchsetzung und beschränken die staatliche Kontrolle

juristischer Personen verzichten und statt formeilnafter Begriffsverweridurig die 'konkreten Interessen eines Kollektivs an der Abwehr von Äußerungen offenlegen. Ausführlich zum Persönlichkeitsschutz von Verbänden Klippel, JZ 1988, 625.

132 Vgl. dazu BVerfGE 97, 125, 148.

133 In diesem Sinne auch Stürner, JZ 1994, 865, 872, der die Aufgabe des Rechts nicht in der Reglementierung von Inhalten, sondern vor allem in „Spielregeln fairer Auseinandersetzung" sieht.

134 Ebenso Findeisen/Hoepner/Zünkler, ZRP 1991, 245, 246.

135 Gounalakis/Rösler (Anm. 45), S. 88.

$136 \mathrm{Vgl}$. zu letzterem oben II.

137 BVerfGE 97, 125, 150 m. w. N. zur Rechtsprechung des BGH. 
daneben auf die Überprüfung der Verhältnismäßigkeit. Da außerdem der Anspruch auf Ersatz der immateriellen Schäden nicht ausschließlich dem Ausgleich dient, sondern durch seine abschreckende Wirkung mindestens ebenso die Vermeidung von Persönlichkeitsverletzungen bezweckt ${ }^{138}$, hat sich auch der Gedanke der Prävention faktisch vom Straf- ins Zivilrecht verlagert (dazu sogleich unten). Die strafrechtliche Sanktion hingegen erweist sich in einer am Verhältnismäßigkeitsgrundsatz orientierten Auswahl von Maßnahmen zum Schutz der Ehre - einmal unterstellt, sie könne diesen wirklich leisten - als Relikt und Fremdkörper. Da sie wegen des Verlusts ihrer präventiven Funktion nicht einmal mehr zur Ergänzung der zivilrechtlichen Rechtsfolgen in schweren Fällen taugt, sollte besser ganz auf sie verzichtet werden.

Die der Demokratie gemäße Reaktion auf eine das Persönlichkeitsrecht verletzende Äußerung ist an erster Stelle der Gegendarstellungsanspruch. Er wurzelt in dem Grundsatz „audiatur et altera pars“ und verleiht dem Angegriffenen Stimme, enthält sich zugleich jedoch des Urteils über den Inhalt oder gar die Wahrheit der ihn hervorrufenden Äußerung ${ }^{139}$. Die inhaltliche Neutralität des Gegendarstellungsrechts reicht erst dann nicht mehr aus, wenn das Interesse des einzelnen berührt ist, ihm fälschlicherweise $\mathrm{Zu}$ gerechnetes abzuwehren, also vor allem bei der Verteidigung gegen unwahre Tatsachenbehauptungen. Den wirksamsten Schutz bietet dabei der Unterlassungsanspruch, der eine Äußerung überhaupt verhindert und somit der Entstehung eines falschen Eindrucks vorbeugt ${ }^{140}$. Ist eine Äußerung dagegen bereits kundgetan, hilft dem Verletzten der Anspruch auf deren Widerruf oder Berichtigung, um so wenigstens die Fortwirkung der Beeinträchtigung einzudämmen ${ }^{141}$. Der Zwang zum Widerruf oder zur Berichtigung der eigenen Äußerung rechtfertigt sich jedoch nur, wenn ihre Unrichtigkeit feststeht, wobei den Verletzten insoweit die Beweislast trifft ${ }^{142}$.

138 So ausdrücklich BGH NJW 1995, 861; NJW 1996, 984; vgl. auch die früheren Entscheidungen BGH NJW 1961, 2059; BVerfG NJW 1973, 1221, 1222; zum Bedeutungsgewinn präventiver und punitiver Elemente innerhalb des Haftungsrechts vgl. Körner, NJW 2000, 241.

139 Dagegen sieht Arzt, JuS 1982, 717, 725, darin eher einen Nachteil des Gegendarstellungsanspruchs.

$140 \mathrm{Vgl}$. zu den Voraussetzungen des Unterlassungsanspruchs Seyfabrt, NJW 1999, 1287, $1288 \mathrm{ff}$.

141 BGH LM \ 1004 BGB, Nr. 54 a; NJW 1995, 861; ausführlich zum Widerrufsanspruch Seyfabrt, NJW 1999, 1287, $1293 \mathrm{f}$.

142 In diesem Sinne BGH NJW 1977, 1681, 1683; ebenso Stürner, JZ 1994, 865, 873; anders Stark (Anm. 9), S. $166 \mathrm{f}$. 
Den größten Bedeutungsverlust hat der strafrechtliche Ehrenschutz durch die Zuerkennung von Schadensersatz bei schweren Persönlichkeitsverletzungen erlitten ${ }^{143}$. Erst ein solcher Entschädigungsanspruch konnte die Lücken im Rechtsschutz der Person schließen, die „Sanktion der Zivilrechtsordnung" hat sich als das "wirksamste und oft einzige Mittel [erwiesen], das geeignet ist, die Respektierung des Personenwerts des Einzelnen zu sichern" 144 . Die tatsächliche Abschreckung durch das Haftungsrecht vermag also gerade vorsätzliche Verletzungen der Rechte einer Person wirksamer zu vermeiden als das Strafrecht ${ }^{145}$. Auch wenn keine astronomischen amerikanischen Phantasiesummen ${ }^{146}$ zugesprochen werden, können drohende Ersatzansprüche, die in ihre Bemessung sowohl die wirtschaftliche Leistungsfähigkeit des Verletzenden als auch die mit der Veröffentlichung erhofften finanziellen Vorteile einbeziehen ${ }^{147}$, gerade auch gegenüber Medienunternehmen verhaltenssteuernd wirken ${ }^{148}$. Dagegen bleiben juristische Personen gegenüber den mahnenden Rufen des Strafrechts taub.

\section{Zusammenfassung und Ausblick}

Die vorstehenden Ausführungen haben gezeigt, daß der Abschaffung der überkommenen Ehrschutzdelikte weder das Verfassungsrecht entgegensteht noch etwaige Schutzlücken zu befürchten sind. Vielmehr hat sich dieses Postulat nur als die rechtspolitisch naheliegende Konsequenz aus kriminalstatistischen Tatsachen sowie straf- und verfassungsrechtlichen Schwächen

143 Grundlegend BGHZ 26, 349; 35, 363, 367f.; vgl. auch BGH NJW 1965, 685, 686; von Caemmerer, Festschrift zum 100jährigen Bestehen des DJT, 1960, S. $49 \mathrm{ff}$.; aus neuer Zeit BGHZ 132, 13, 27 ff.; BGH NJW 1995, 861; NJW 1996, 984; Gounalakis/Rösler (Anm. 45), S. 97 ff.; Körner, NJW 2000, 241, 244.

144 Fast wortgleich BGHZ 35, 363, 368; BVerfG NJW 1973, 1221, 1222; BGH NJW 1995, 861, 864f.; NJW 1996, 984, 985; BVerfG NJW 2000, 2187.

145 Kritisch gegenüber dieser Zurückdrängung des Strafrechts Pfeifer, JR 1996, 420, 422.

146 Vgl. zum amerikanischen Recht Kretschmer, Strafrechtlicher Ehrenschutz und Meinungs- und Pressefreiheit im Recht der Bundesrepublik Deutschland und der Vereinigten Staaten von Amerika, 1994, S. $89 \mathrm{ff}$.; Stürner, JZ 1994, 865, $868 \mathrm{ff}$.; Timm, Schutz von Amtsträgern vor diffamierenden Äußerungen in der Presse in Deutschland und in den USA, 1999, S. $166 \mathrm{ff} ., 183$ m. w. N.

147 Zur Bemessung der Höhe des Anspruchs Prinz, NJW 1996, 953, 954 ff.; Schlechtriem, JZ 1995, 362, $363 \mathrm{f}$.

148 Körner, NJW 2000, 241, 244; vgl. auch Diederichsen, AcP 182 (1982), S. 101, 111 f.; Magnus, Schaden und Ersatz, 1987, S. 282; Rosengarten, NJW 1996, 1935, 1937. 
der Beleidigungstatbestände erwiesen. Wenn auch jeder einzelne der vorgebrachten Einwände je nach Tatbestand bald stärker, bald schwächer erscheint, nehmen doch die dargestellten Mängel in ihrer Gesamtheit dem strafrechtlichen Ehrenschutz seine Berechtigung - die Waffe des Strafrechts ist auf dem Feld der Ehre zu grob und zugleich zu stumpf. Wollte man aus rechtspolitischen Überlegungen Amtsträgern einen besonderen Ehrenschutz zuteil werden lassen, so ließe sich dies zwar mit den über die Ehre hinausgreifenden Rechtsgütern des Amtes, der Funktion oder der Institution begründen (vgl. etwa $\$ 99 \mathrm{StGB}$ ) oder auch auf Fälle beschränken, in denen die Ehrverletzung den öffentlichen Frieden bedroht (vgl. $\int S$ 166, 130 StGB); auch solche Sondertatbestände sollte man jedoch im Hinblick auf Art. 5 GG nur mit Vorsicht in Erwägung ziehen. Was den allgemeinen strafrechtlichen Ehrenschutz, jedenfalls aber die Beleidigung betrifft, so sollte der Gesetzgeber den Mut aufbringen, die Tendenzen zur Liberalisierung und Deregulierung der letzten Jahre in das Strafrecht zu tragen. Weder droht dem - auch nicht-prominenten - Bundesbürger der Verlust seiner Ehre durch eine Verrohung der Sitten, noch sollte die Rechtspolitik seine Ehre dadurch in Frage stellen, daß sie ihm eine wirksame Verteidigung derselben auf dem Zivilrechtsweg nicht zutraut. Mehr Freiheit und Eigenverantwortung darf durchaus gewagt werden. 